\title{
Squamous cell carcinoma of the esophagus with mucin-secreting component
}

\author{
Mucoepidermoid carcinoma
}

\begin{abstract}
Among 1058 patients with cancer of the esophagus, 20 patients with mucoepidermoid or adenosquamous cell carcinoma of the esophagus and cardia, together defined as squamous cell carcinoma with a mucin-secreting component, were seen over a 10 -year period. Their records were reviewed and appropriate comparisons were also made with the more common squamous cell carcinomas and adenocarcinomas. Squamous cell carcinoma with mucin-secreting component comprised $1.9 \%$ of all tumors encountered. Clinical features including age, male predominance, symptoms at presentation, length of tumor, and appearance of tumor did not differ from those of squamous cell and adenocarcinoma. The location of these tumors, however, followed that of squamous cell carcinomas, with $55 \%$ in the middle third and $25 \%$ in the lower third. Adenocarcinomas were found predominantly at the cardia $(\mathbf{8 3} \%)$. Operability and resectability rates were higher than those of squamous cell and adenocarcinomas. Primary treatment consisted of resection in 19 of the 20 patients $(95 \%)$; 18 of them had a one-stage resection and 1 patient had a two-stage resection. Postresection staging showed that $5 \%$ had stage I disease, $16 \%$ had stage II, and $79 \%$ had stage III disease. None of the patients who underwent resection died within 30 days of the operation. The mortality after 30 days was $10.5 \%$. The 1 patient in whom intubation was the primary treatment had distant metastases at the time of presentation (stage IV). The overall median survival was 9.2 months. The median survival for patients who had their tumors resected was 9.5 months. The survival improved to 33 months for curative resection but was only 8.7 months for palliative resection. The 1-, 2-, and 5-year survivals were $46 \%$, $39 \%$, and $0 \%$, respectively. This prognosis was not significantly different from that of patients with squamous cell carcinoma or adenocarcinoma. (J THORAC CARDIOvasC SuRG 1994;107:62-7)
\end{abstract}

Elliott Fegelman, MD, ${ }^{\mathrm{a}}$ Simon Y. K. Law, FRCSEd, MA, ${ }^{\mathrm{a}}$ Manson Fok, FRCSEd, ${ }^{\mathrm{a}}$

K. Y. Lam, MB, BS, ${ }^{b}$ S. L. Loke, MRCPath, ${ }^{b}$ L. T. Ma, PhD, FRCPA, ${ }^{\text {b }}$ and

John Wong, PhD, FRACS, FACS, ${ }^{a}$ Hong Kong

8 Oquamous cell carcinoma of the esophagus with mucin-secreting component is a rare primary tumor of the esophagus, whose nomenclature has been confusing. Terms like mucoepidermoid carcinoma and adenosquamous carcinoma have been used most frequently in

From the Departments of Surgery ${ }^{\mathrm{a}}$ and Pathology, ${ }^{\mathrm{b}}$ The University of Hong Kong, Queen Mary Hospital, Hong Kong.

Received for publication Feb, 1, 1993.

Accepted for publication March 22, 1993.

Address for reprints: John Wong, PhD, FRACS, FACS, Department of Surgery, The University of Hong Kong, Queen Mary Hospital, Hong Kong.

Copyright $\odot 1994$ by Mosby-Year Book, Inc.

$0022-5223 / 94 \$ 1.00+.10 \quad 12 / 1 / 47950$

its description. It is a mixed-cell tumor with both adenocarcinomatous and squamous components, and it is believed to arise from the submucosal salivary glands of

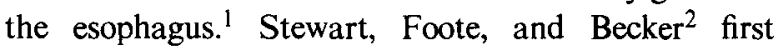
described "mucoepidermoid carcinoma" in the minor salivary glands of the head and neck in 1945, and this tumor type is now widely recognized in this region. Since that time, histologically similar salivary glands have been identified throughout the esophagus, but primary carcinoma of these glands remains rare. From this relatively small experience, comments regarding its presentation, operability, and prognosis have necessarily been speculative and range from better ${ }^{3,4}$ to worse $e^{5,6}$ than that of the more common squamous cell carcinoma of the esophagus.

This review will focus on the 20 patients with squamous 


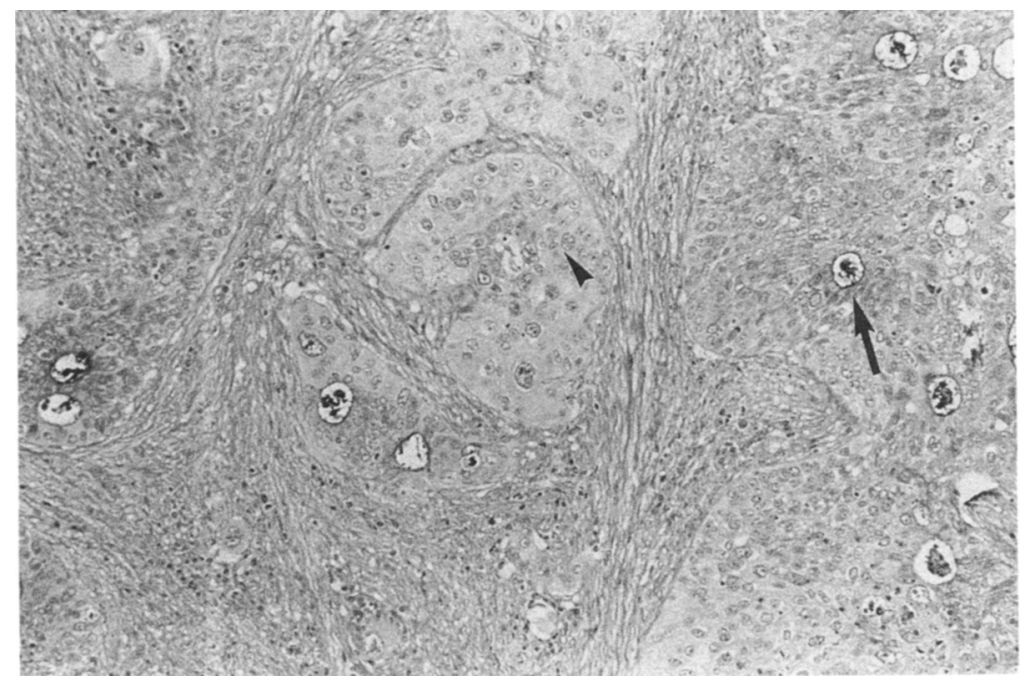

Fig. 1. Squamous (arrowhead) and adenomatous components (arrow) in the carcinoma are identified. (Hematoxylin and eosin stain; original magnification $\times 120$.)

cell carcinoma of the esophagus with mucin-secreting component seen in the Department of Surgery, The University of Hong Kong, at the Queen Mary Hospital over the past 10 years. We have previously compared our results of surgical resection for patients with squamous cell carcinoma and adenocarcinoma. ${ }^{7}$ In this review, comparisons are also made with the more common squamous cell and adenocarcinomas where appropriate.

\section{Patients and method}

Between October 1981 and April 1992, 1058 patients with esophageal cancers were referred to the Department of Surgery, The University of Hong Kong, at the Queen Mary Hospital. These patients underwent similar diagnostic workup, including complete history and physical examination, barium swallow, examination with a flexible esophagoscope and bronchoscope, chest roentgenography, abdominal ultrasonography, and biochemical and nutritional analysis. Computed tomographic scan was not performed routinely to evaluate extraesophageal extension of disease because of limited availability. From this database 20 patients with histologically confirmed squamous cell carcinoma with mucin-secreting component of the esophagus were identified (Fig. 1). The histologic sections were reviewed by three of the authors (Lam, Loke, and $\mathrm{Ma}$ ) and the presence of mucin-secreting component was confirmed by mucin stain (mucicarmine). In adenosquamous carcinoma, the adenocarcinomatous component is usually tubular and the squamous and adenomatous carcinomatous components are more clear-cut. In mucoepidermoid carcinoma, the squamous component is usually beneath the mucus-secreting epithelium. However, the distinction between these two entities was not possible in all cases. Thus they were analyzed as a group in this study. The symptom complex, operative and nonoperative intervention, outcome, and survival were evaluated for this subtype of esophageal cancer.

When tumor was resected, the resection was curative if the tumor was confined to the esophagus and the adjacent lymph nodes either were uninvolved or were less than $2 \mathrm{~cm}$ in diameter, discrete, and within the vicinity of the primary tumor. Both the tumor and the adjacent lymph nodes were removed completely. Resection was palliative when there was tumor infiltration beyond the esophagus into mediastinal organs, with gross lymph node metastases, and when there was actual or a strong likelihood of tumor being left behind after resection.

Patients were followed-up every second week for 2 months, monthly thereafter for 1 year, and then at intervals of 3 months. Statistical differences were determined by the $\chi^{2}$ test or Fisher's exact test when the numbers were small. Group differences were regarded as significant when $p$ was less than 0.05 .

\section{Results}

Of the 1058 patients, $20(1.9 \%)$ had squamous cell carcinomas with mucin-secreting component, squamous cell carcinomas were found in 816 patients $(77.1 \%)$, and adenocarcinomas were seen in 181 patients $(17.1 \%)$. Small-cell carcinomas were found in 11 patients $(1 \%)$ and other rare tumors in the remaining 23 patients $(2.2 \%)$. The histologic classification of the primary tumor was unknown in 7 patients $(0.7 \%)$.

The mean age of patients with squamous cell carcinoma with mucin-secreting component at the time of presentation was $65.7 \pm 9.4$ years, not significantly different from those of patients with squamous cell carcinoma $(63.4 \pm 9.5$ years $)$ or adenocarcinoma $(65.4 \pm 10.7$ years). Eighteen of the 20 patients were male, for a male/female ratio of 9:1. Male predominance was similar in patients with squamous cell carcinoma (5.8:1) and adenocarcinoma (3.2:1).

The main presenting symptom in most cases of esophageal cancer is dysphagia. Nineteen patients $(95 \%)$ 


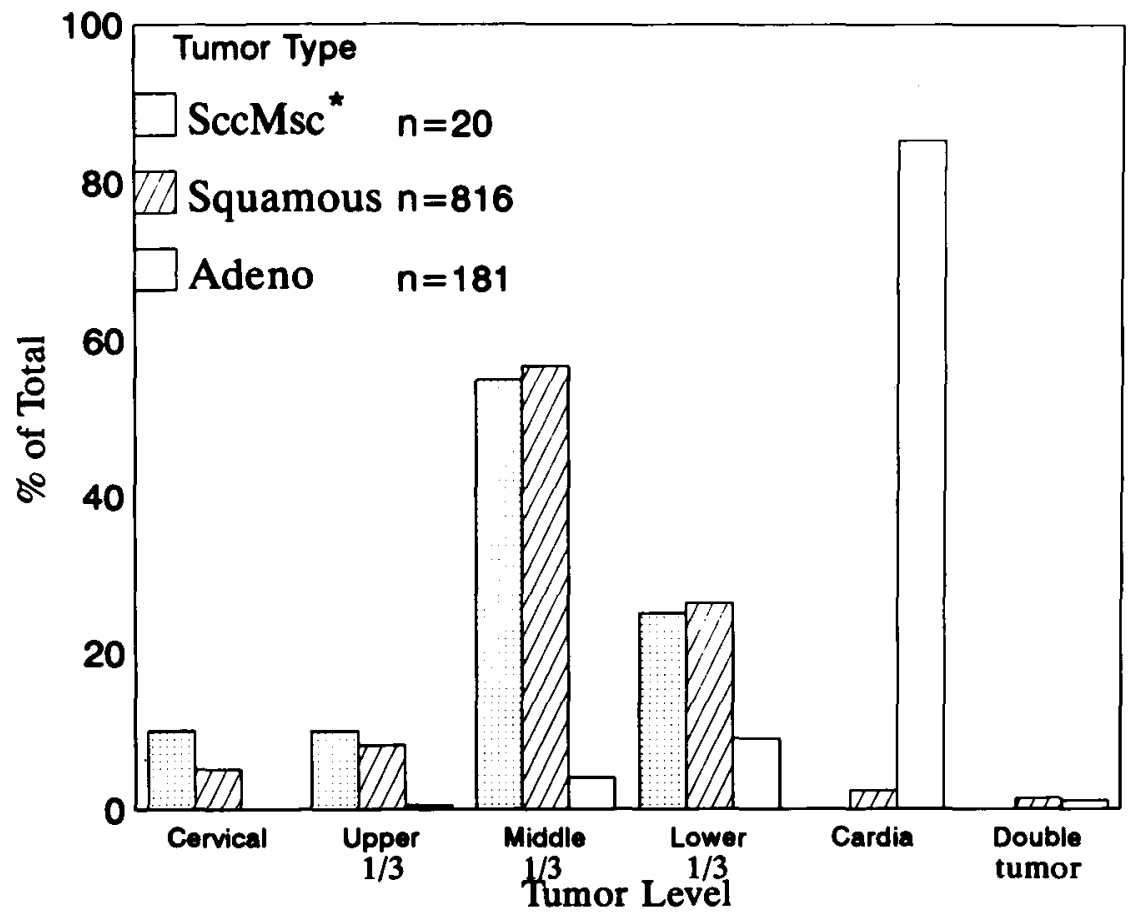

Fig. 2. Level of primary tumor. ${ }^{*}$ Squamous cell carcinoma with mucin-secreting component.

initially complained of dysphagia, and one had a neck mass. The mean time between onset of symptoms and presentation was $7.2 \pm 5.9$ weeks, not significantly different from that of patients who had squamous cell carcinoma $(9.1 \pm 9.6$ weeks $)$ or adenocarcinoma (12.5 \pm 14.7 weeks).

The distribution of squamous cell carcinoma with mucin-secreting component along the length of the esophagus paralleled that of squamous cell carcinoma rather than that of adenocarcinomas (Fig. 2). The tumors were found in the middle third of the esophagus in $11(55 \%)$ of the 20 patients, in the lower third in $5(25 \%)$, in the upper third in $2(10 \%)$, and in the cervical esophagus in $2(10 \%)$.

The length of the primary squamous cell carcinoma with mucin-secreting component, as measured before the operation with a flexible endoscope or by barium swallow, was $6.1 \pm 3.2 \mathrm{~cm}$ and was not significantly different from the length of either squamous cell carcinomas $(5.8 \pm 2.9$ $\mathrm{cm})$ or adenocarcinomas $(6.0 \pm 4.6 \mathrm{~cm})$.

Accurate histologic diagnosis was possible by preoperative endoscopic biopsy in only 4 of the 20 patients ( $20 \%$ ). In all but 1 patient a diagnosis of squamous cell carcinoma was reported and subsequently revised to squamous cell carcinoma with mucin-secreting component when the whole resected specimen was examined. The last patient's biopsy specimen showed cellular atypia only.

Operability rates were high for all three types of tumors
(Table I). Only 1 ( $5 \%$ ) of our 20 patients with squamous cell carcinoma with mucin-secreting component had intubation as the primary treatment because of carcinomatosis. Nineteen $(95 \%)$ underwent an operation. This rate was significantly higher than the operability rates for patients with squamous cell carcinoma (76.2\%, $p=0.001)$ or adenocarcinoma $(74 \%, p=0.01)$. Resectability rates were also significantly higher $(95 \%)$ than for squamous cell carcinoma $(61 \%, p<0.01)$ or for adenocarcinoma $(60 \%, p<0.01)$ (Table I). Once resection was selected as the mode of treatment, the proportions of curative resection were similar at around $40 \%$ for all three types of tumors (Table II).

The type of procedure performed depended on the location of the tumor, its extent, and also the cardiorespiratory reserve of the patient. The commonest procedure performed was a Lewis-Tanner esophagectomy, with the stomach used for reconstruction. The technique has been described in our previous report. ${ }^{8}$ Ten patients (53\%) underwent this procedure. Other operative interventions included three-phase esophagectomy in 3 patients for tumor above the aortic arch, transhiatal esophagectomy in 2 patients, pharyngolaryngoesophagectomy in 2 patients, esophagogastrectomy in 1 patient, and two-stage operation with esophagectomy followed by retrosternal gastric reconstruction in 1 patient.

Table III demonstrates staging of resected tumors as based on intraoperative findings and pathologic assess- 
Table I. Main treatment given to 1017 patients with esophageal cancer*

\begin{tabular}{|c|c|c|c|c|c|c|}
\hline & \multicolumn{2}{|c|}{ SccMsct } & \multicolumn{2}{|c|}{ Squamous } & \multicolumn{2}{|c|}{ Adenocarcinoma } \\
\hline & $\overline{N o}$ & $\%$ & No. & $\%$ & No. & $\%$ \\
\hline Operation & 19 & 95.0 & 622 & 76.2 & 134 & 74.0 \\
\hline Resection & 19 & 95 & 501 & 61 & 108 & 60 \\
\hline Intubation & 1 & 5.0 & 21 & 2.6 & 6 & 3.3 \\
\hline Radiotherapy & - & - & 33 & 4.1 & - & - \\
\hline No intervention & - & - & 117 & 14.3 & 30 & 16.6 \\
\hline Other & - & - & 23 & 2.8 & 11 & 6.1 \\
\hline Total & 20 & 100 & 816 & 100 & 181 & 100 \\
\hline
\end{tabular}

*Other pathologic types were excluded.

†Squamous cell carcinoma with mucin-secreting component.

Table II. Types of operation performed

\begin{tabular}{|c|c|c|c|c|c|c|}
\hline & \multicolumn{2}{|c|}{$S c c M s c^{*}$} & \multicolumn{2}{|c|}{ Squamous } & \multicolumn{2}{|c|}{ Adenocarcinoma } \\
\hline & No. & $\%$ & No. & $\%$ & No. & $\%$ \\
\hline Resected & 19 & 100 & 501 & 80.5 & 108 & 80.6 \\
\hline Curative & 8 & 42 & 200 & 40 & 48 & 44 \\
\hline Palliative & $I I$ & 58 & 301 & 60 & 60 & 56 \\
\hline Bypass & - & - & 110 & 17.7 & 9 & 6.7 \\
\hline Explored only & - & - & 11 & 1.8 & 17 & 12.7 \\
\hline Total & 19 & 100 & 622 & 100 & 134 & 100 \\
\hline
\end{tabular}

*Squamous cell carcinoma with mucin-secreting component.

ment. Staging method is based on the American Joint Committee on Cancer (AJCC) classification, 1988. ${ }^{9}$ Overall, stage III disease predominated in all three groups, comprising $79 \%$ of squamous cell carcinomas with mucin-secreting component and $70 \%$ and $58 \%$ of squamous and adenocarcinomas, respectively. Stage IV disease was uncommon in our series of patients with squamous cell carcinoma with mucin-secreting component. The only patient who had stage IV disease was not operated on and had intubation as the primary treatment. Overall, staging distribution was not significantly different among the three types of tumors.

None of the patients who underwent resection died within 30 days of the operation. Two (10.5\%) patients died in the hospital but more than 30 days after the operation: one died of aspiration pneumonia and the other of anastomotic leakage. Patients with squamous cell carcinoma had a $4.7 \%$ 30-day operative mortality, and $10.7 \%$ of patients died more than 30 days after the operation. Patients with adenocarcinoma had a 6.8\% 30-day mortality and a $3.8 \%$ mortality after 30 days.

Overall prognosis was not significantly different among the three types of tumors. The median survival of patients with squamous cell carcinoma with mucin-secreting component from the time of presentation was 9.2 months, not different from patients with squamous cell carcinoma at 7 months $(p=0.51)$ or from those with adenocarcino-
Table III. Staging of resected tumors

\begin{tabular}{lccc}
\hline & $\begin{array}{c}\text { SccMsc* } \\
(\%)\end{array}$ & $\begin{array}{c}\text { Squamous } \\
(\%)\end{array}$ & $\begin{array}{c}\text { Adenocarcinoma } \\
(\%)\end{array}$ \\
\hline Stage I & 5 & 2 & 2 \\
Stage II & 16 & 21 & 15 \\
Stage III & 79 & 70 & 58 \\
Stage IV & 0 & 7 & 25 \\
\hline
\end{tabular}

*Squamous cell carcinoma with mucin-secreting component.

mas at 9.1 months $(p=0.41)$. For the 19 patients who had resection, the median survival was 9.5 months (Table IV). Patients with either squamous cell carcinoma or adenocarcinoma had a median survival of 10.4 months. In the 8 patients who underwent curative resection, the median survival was 33 months, and it was 8.7 months for the 11 patients who underwent palliative resection. The median survivals for patients with squamous cell carcinomas and adenocarcinomas who underwent curative resection were 24.4 months and 17.5 months, respectively, and were 6.6 months and 8.7 months, respectively, after palliative resection.

Of the original 20 patients in this review, $14(70 \%)$ have died and $6(30 \%)$ are alive at a mean of 17 months, 5 of them without evidence of tumor recurrence. There have not yet been any 5 -year survivors. The primary cause of death was related to malignancy in 6 patients $(43 \%)$ and 
Table IV. Survival in patients with tumors resected

\begin{tabular}{|c|c|c|c|c|c|c|c|}
\hline & \multicolumn{2}{|c|}{$S c c M s c^{*}$} & \multicolumn{2}{|c|}{ Squamous } & \multicolumn{3}{|c|}{ Adenocarcinoma } \\
\hline & Curative & Palliative & Curative & Palliative & Curative & & Palliative \\
\hline $\begin{array}{l}\text { Median survival } \\
\text { (mo) }\end{array}$ & 33.0 & 8.7 & 24.4 & 6.6 & 17.5 & & 8.7 \\
\hline $\begin{array}{l}\text { Overall median survival } \\
\quad(\mathrm{mo})\end{array}$ & \multicolumn{2}{|c|}{9.5} & \multicolumn{2}{|c|}{10.4} & \multicolumn{3}{|c|}{10.4} \\
\hline \multicolumn{8}{|l|}{ Survival (\%) } \\
\hline One year & 75 & 16 & 68 & 26 & 58 & & 32 \\
\hline Two year & 60 & 16 & 50 & 11 & 41 & & 7.5 \\
\hline Five year & 0 & 0 & 31 & 7 & 38 & & 0 \\
\hline \multicolumn{8}{|l|}{ Overall survival (\%) } \\
\hline One year & \multicolumn{2}{|c|}{$\begin{array}{l}46 \\
30\end{array}$} & \multicolumn{2}{|c|}{42} & \multicolumn{3}{|c|}{43} \\
\hline Two year & \multirow{2}{*}{\multicolumn{2}{|c|}{$\begin{array}{r}39 \\
0\end{array}$}} & \multirow{2}{*}{\multicolumn{2}{|c|}{$\begin{array}{l}26 \\
17\end{array}$}} & \multicolumn{3}{|c|}{21} \\
\hline Five year & & & & & & 17 & \\
\hline
\end{tabular}

*Squamous cell carcinoma with mucin-secreting component.

respiratory complications in $3(21 \%)$. There was 1 death (7\%) caused by anastomotic leak, $1(7 \%)$ caused by tracheobronchial recurrence, and $1(7 \%)$ caused by neck recurrence. The cause of death was unknown in the remaining 2 patients (14\%).

\section{Comment}

Previous reviews of literature on esophageal squamous cell carcinoma with a mucin-secreting component consisted of only small numbers of cases. ${ }^{6,10}$ The small numbers may be due to underestimation of reported cases by the authors. The confusing nomenclature used to describe these mixed-cell tumors has also contributed to the underreporting. Squamous cell carcinoma with mucinsecreting component has in turn been identified as "mucoepidermoid carcinoma, adenoacanthoma, adenocarcinoma with squamous metaplasia, mixed squamous and glandular carcinoma, adenosquamous carcinoma...." A recent review of the literature from the Department of Pathology, The University of Hong Kong, revealed 74 cases in the Japanese literature and 114 cases in the Western literature (unpublished data). Most reports, however, were based on small numbers of patients. Each author concentrated more on anatomic and histologic descriptions of the tumor, rather than on evaluating its natural history or response to treatment. ${ }^{1,3,4,11-13}$ In this study, we analyzed the clinical features of these tumors in a descriptive approach by using the term squamous cell carcinoma with mucin-secreting component to include both the mucoepidermoid and adenosquamous carcinomas, because the two tumor types have similar features.

On review of patients with this type of tumor, most clinical features were not appreciably different from those of squamous cell carcinoma or adenocarcinoma. The disease affects predominantly men during the sixth decade of life. The majority (95\%) present with the sine qua non of esophageal cancers-dysphagia. The duration of symptoms, however, cannot be used to distinguish among the different types of tumors because the difference is small and the variation in time to presentation is substantial.

Neither the position of the tumor within the esophagus nor its appearance at the time of endoscopy could determine the correct histologic characteristics of this tumor. However, when squamous cell carcinoma with mucin-secreting component and squamous cell carcinoma were compared with adenocarcinomas, $83 \%$ of the latter tumor were found at the cardiac region of the stomach, with less than $15 \%$ located at the middle and lower thirds of the esophagus. In contrast to patients in Western countries, the rarity of Barrett's esophagus and reflux diseases in Asians may partly account for the higher proportion of adenocarcinoma involving the lower third of the esophagus in the white population. ${ }^{7}$ Bell-Thomson, Haggitt, and Ellis ${ }^{11}$ suggested that because mucoepidermoid carcinomas originate in the submucosal glands of the esophagus, the original barium swallow roentgenogram might have a smoother appearance and thus help to distinguish this tumor type. We did not find this to be so, however, probably because of the late stage of presentation.

The operability and resectability rates of squamous cell carcinoma with mucin-secreting component were significantly higher than those of the other two types of tumors, a fact which suggests that this type of tumor may become apparent at an earlier stage. However, there may be bias in this conclusion because of the difficulty in obtaining representative biopsy specimens showing both the squamous and glandular elements. Eighty percent of the biopsy specimens were reported as squamous cell carcinoma only, until the resected esophagus was examined. Patients with advanced disease who had not undergone 
operation and resection nor had postmortem examination would have been misclassified as having squamous cell carcinoma and thus became lost in the analysis. This misclassification would have increased the operability and resectability rates. It also may help to explain the paucity of stage IV disease for this type of tumor.

The location of the primary tumor and its degree of local tissue involvement determined what the operative approach would be. Because few of our patients underwent preoperative computed tomography unless there was clear evidence of distant spread before the operation, the decision to resect or bypass the tumor was usually determined before the operation and confirmed during the operation. In addition, the degree of local tumor involvement was assessed during exploration and dissection. There was no statistically significant difference in the gross evaluation of local spread of disease by the operating surgeon among the three types of tumors. Most patients had stage III disease.

Previous authors have speculated about the aggressiveness of squamous cell carcinoma with mucin-secreting component. Sasajima, ${ }^{6}$ Osamura, ${ }^{5}$ and their associates suggested that the disease was more aggressive than squamous cell carcinoma, whereas Weitzner ${ }^{4}$ and Kay, ${ }^{3}$ admitting to too few cases to make any real judgment, believed it to be less aggressive. Bell-Thomson, Haggitt, and Ellis ${ }^{11}$ have concluded that the survival of patients with this disease was not different from that of patients with squamous cell carcinoma. This review confirms that resected squamous cell carcinomas with mucin-secreting component have no statistically significant differences in survival compared with the other more common tumors. When survival statistics were evaluated at 1,2 , and 5 years for each tumor type, patients with mucoepidermoid carcinoma appeared to do better initially (Table IV). Except for patients undergoing a palliative resection of their tumor, a greater (but not statistically significant) percentage of patients with squamous cell carcinoma with mucin-secreting component were alive at 1 and 2 years. However, a 4-year survival has been the longest. Not even those patients undergoing curative resections have survived more than 48 months, whereas there was a $17 \%$ 5 -year survival for patients undergoing resection of squamous cell or adenocarcinomas. This survival improved to $31 \%$ for patients with curative resections of squamous cell carcinoma and $38 \%$ for patients with curative resections of adenocarcinoma.

In this review of squamous cell carcinoma with mucinsecreting component seen over a 10 -year period, these cancers did not differ significantly in presentation, degree of tumor extension, or response to surgical therapy from either squamous cell tumors or adenocarcinomas. The only difference was that their location tended to conform to that of squamous tumors rather than that of adenocarcinomas. There have been no 5-year survivors among patients with this cancer during the 10 years of this review.

\section{REFERENCES}

1. Karaki Y, Katoh H, Shimazaki K, et al. Histogenesis of adenosquamous carcinoma of the esophagus. In: Siewert JR, Holscher AH, eds. Diseases of the esophagus. Berlin: Springer-Verlag, 1988:60-3.

2. Stewart FW, Foote FW, Becker WF. Mucoepidermoid tumors of salivary glands. Ann Surg 1945;122:820-44.

3. Kay S. Mucoepidermoid carcinoma of the esophagus: report of two cases. Cancer 1968;22:1053-9.

4. Weitzner S. Mucoepidermoid carcinoma of esophagus: report of a case. Arch Pathol 1970;90:271-3.

5. Osamura RY,Sato S, Miwa M, Miwa T. Mucoepidermoid carcinoma of the esophagus: report of an unoperated autopsy case and review of literature. Am J Gastroenterol 1978;69:467-70.

6. Sasajima K, Watanabe M, Takubo K, Takai A, Yamashita K, Onda M. Mucoepidermoid carcinoma of the esophagus: report of two cases and review of literature. Endoscopy 1990;22:140-3.

7. Law SYK, Fok M, Cheng SWK, Wong J. A comparison of outcome after resection for squamous cell carcinomas and adenocarcinomas of the esophagus and cardia. Surg Gynecol Obstet 1992;175:107-12.

8. Wong J. Stapled esophagogastric anastomosis in the apex of the right chest after subtotal esophagectomy for carcinoma. Surg Gynecol Obstet 1987;164:568-72.

9. Beahrs OH, Henson DE, Hutter RVP, Myers MH. Manual for staging of cancer. 3rd edition. American Joint Committee on Cancer. Philadelphia: JB Lippincott, 1988.

10. Ozawa S, Ando N, Shinozawa Y, et al. Two cases of resected esophageal mucoepidermoid carcinoma. Jpn J Surg 1989;19:86-92.

11. Bell-Thomson J, Haggitt RC, Ellis FH Jr. Mucoepidermoid and adenoid cystic carcinomas of the esophagus. J Thorac Cardiovasc SURG 1980;79:438-46.

12. Kuwano H, Ueo H, Sugimachi K, et al. Glandular or mucus-secreting components in squamous cell carcinoma of the esophagus. Cancer 1985;56:514-8.

13. Kuwano H, Matsuda H, Koga $Y$, et al. Glandular or mucus-secreting components in squamous cell carcinoma of the esophagus. In: Siewert JR, Holscher AH, eds. Diseases of the esophagus. Berlin: Springer-Verlag, 1988:64-6. 